\title{
Osteal Macrophages and Megakaryocytes Increase Adipogenesis
}

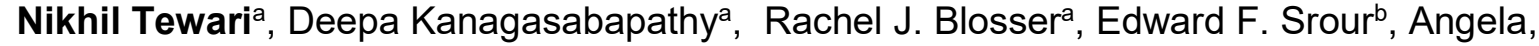
Bruzzanitic, Melissa A. Kacena ${ }^{a}$

Departments of arthopaedic Surgery and ${ }^{b}$ Medicine, Indiana University School of Medicine, Indianapolis, IN, USA

'Department of Biomedical Sciences \& Comprehensive Care, Indiana University School of

Dentistry, Indianapolis, IN, USA

Bone marrow adipose tissue (MAT) increases with aging and contributes to low bone density and skeletal fractures. However, the cells and factors within the bone marrow (BM) that regulate adipogenesis remain poorly understood. In the current study, we examined the role of osteal macrophages (OMs) and megakaryocytes (MKs) on the regulation of adipogenesis. We cultured murine osteoblasts/osteoblast progenitors (OBs from hereon) derived from neonatal calvarial cells (CCs, a combination of OBs and OMs) or OBs isolated by fluorescence activated cell sorting (FACS) in the presence or absence of fetal liver derived murine MK. The cells underwent induced adipogenesis for 5-7 days by supplementation of media with insulin, indomethacin, and dexamethasone, and then the number of adipocytes was quantified. We found that co-culturing MKs and OMs with OBs results in up to a 7.8-fold and 11.7-fold increase in adipocytes, respectively. We also elucidated that thrombopoietin (TPO), the major growth factor for MKs, inhibits adipogenesis in both OBs and CCs by approximately $60 \%$. Similarly, we found that CCs and OBs derived from mice deficient in the TPO receptor, Mpl, had approximately $30 \%$ more adipocytes than their wild-type (WT) counterparts. Finally, in vitro findings were corroborated in vivo through quantification of MKs and adipocytes in mice in which MK number was elevated or reduced. Mice with significantly higher numbers of BM-residing MKs also had significantly higher numbers of BM-residing adipocytes. Because there is typically an inverse relationship between adipogenesis and osteogenesis, understanding ways to inhibit adipogenesis could lead to an increase in $\mathrm{OB}$ number and bone formation, which in turn could lead to new treatments for bone loss diseases such as osteoporosis. 